\title{
Selenato e selenito na produção e biofortificação agronômica com selênio em arroz
}

\author{
Paulo Fernandes Boldrin(1), Valdemar Faquin(1), Sílvio Júnio Ramos(1), Luiz Roberto Guimarães Guilherme(1), \\ Carla Elisa Alves Bastos ${ }^{(2)}$, Geila Santos Carvalho( ${ }^{(1)}$ e Enio Tarso de Souza Costa(1)
}

\begin{abstract}
(1)Universidade Federal de Lavras, Departamento de Ciência do Solo, Caixa Postal 3037, CEP 37200-00 Lavras, MG. E-mail: pboldrin@ig.com.br, vafaquin@dcs.ufla.br, silviojramos@dcs.ufla.br, guilherm@dcs.ufla.br, geilacarvalho@gmail.com.br, eniotsc@gmail.com (2)Universidade de São Paulo, Escola Superior de Agricultura Luiz de Queiroz, Departamento de Ciência do Solo, Caixa Postal 9, CEP 13418-900 Piracicaba, SP. E-mail: ceabastos@usp.br
\end{abstract}

Resumo - O objetivo deste trabalho foi avaliar o efeito da aplicação de doses de selenato e selenito na biofortificação de arroz (Oryza sativa) com Se, bem como a influência dessas formas de Se nos teores de P, S, $\mathrm{Fe}$ e $\mathrm{Zn}$ nos grãos. $\mathrm{O}$ experimento foi conduzido em casa de vegetação, em vasos com $4 \mathrm{dm}^{3}$ de um Latossolo Vermelho-Amarelo distrófico, de textura média, em arranjo fatorial $5 \times 2$, com cinco doses de $\mathrm{Se}(0,0,75,1,50$, 3,0 e $6,0 \mathrm{mg} \mathrm{dm}^{-3}$ ) e duas formas de Se (selenato e selenito). Observou-se que o selenato proporcionou maior eficiência na absorção de Se pelas raízes, aproveitamento pela planta, translocação para a parte aérea e teor desse elemento nos grãos. A aplicação do selênio na adubação altera os teores de $\mathrm{P}, \mathrm{S}$ e $\mathrm{Zn}$, mas não afeta os de Fe nos grãos de arroz.

Termos para indexação: arroz enriquecido, aproveitamento de selênio, segurança alimentar, selenato, selenito.

\section{Selenate and selenite on yield and agronomic biofortification with selenium in rice}

\begin{abstract}
The objective of this work was to evaluate the effect of doses of selenate and selenite on rice (Oryza sativa) biofortification with $\mathrm{Se}$, as well the influence of these forms of Se in the levels of $\mathrm{P}, \mathrm{S}, \mathrm{Fe}$, and $\mathrm{Zn}$ in grains. The experiment was conducted in a greenhouse, in pots with $4 \mathrm{dm}^{3}$ of a sandy clay loam Latosol, with medium texture, in a $5 \times 2$ factorial arrangement with five doses of Se $\left(0,0.75,1.50,3.0\right.$, and $\left.6.0 \mathrm{mg} \mathrm{dm}^{-3}\right)$ and two forms of Se (selenate and selenite). Selenate provided greater efficiency of root uptake of Se, plant-use efficiency, translocation from roots to shoots, and content of this element in rice grains. The application of Se during fertilization influences the levels of $\mathrm{P}, \mathrm{S}$, and $\mathrm{Zn}$, but does not affect those of $\mathrm{Fe}$ in rice grains.
\end{abstract}

Index terms: enriched rice, use of selenium, food security, selenate, selenite.

\section{Introdução}

O selênio (Se) é considerado essencial para os seres humanos e animais (Rayman, 2002). Em diversas regiões do mundo, a baixa ingestão dietética desse elemento tem sido associada a problemas de saúde humana, como aumento na incidência de câncer (Combs, 2001). Uma das estratégias para diminuir a deficiência de Se tem sido aumentar o teor desse elemento nos alimentos (White \& Broadley, 2009; Ramos et al., 2010).

A biofortificação agronômica com Se, que consiste basicamente no aumento da concentração desse elemento nas culturas agrícolas, por meio da sua introdução na adubação das plantas, tem mostrado resultados satisfatórios (Graham et al., 2007). Chen et al. (2002) verificaram que o teor de Se na cultura do arroz aumentou com a introdução desse elemento na adubação, o que indica que esta técnica é útil para elevar a ingestão de Se pela população. Além disso, Combs (2001) relatou que o nível adequado de Se em uma população está altamente correlacionado ao conteúdo de Se nos alimentos. Portanto, ao se considerar que o arroz é o alimento básico predominante em mais de 30 países (Lucca et al., 2006) e que a deficiência do Se atinge aproximadamente um bilhão de pessoas no mundo (White \& Broadley, 2009), essa cultura agrícola apresenta enorme potencial para estudos que visem diminuir a deficiência de Se na população mundial. Entretanto, no Brasil, há poucos estudos deste tipo, embora haja indícios de baixa ingestão de Se pela população (Ferreira et al., 2002; Maihara et al., 2004).

Cabe destacar que, nos programas de biofortificação com Se, deve-se levar em conta a forma de $\mathrm{Se}$ 
aplicada, uma vez que os solos intemperizados, com elevadas concentrações de óxidos de $\mathrm{Fe}$ e $\mathrm{Al}$ na fração argila, podem adsorver selenito, o que reduz, consequentemente, sua disponibilidade para as plantas (Zhang \& Sparks, 1990). Segundo Rovira et al. (2008), o Se na forma de selenito pode sofrer adsorção específica com a hematita e a goethita e formar complexos de esfera-interna, o que pode torná-lo indisponível para as plantas. $\mathrm{O}$ mesmo não ocorre com o Se na forma de selenato, que é estável em ambientes oxidados, muito móvel no solo e está prontamente disponível para as plantas. Além das diferenças de disponibilidade das formas de Se no solo, essas também diferem na absorção e na mobilidade do Se no interior das plantas, em que o Se do selenato é mais facilmente transportado para a parte aérea, enquanto o Se do selenito tende a acumular nas raízes das plantas (Zhang et al., 2003).

O objetivo deste trabalho foi avaliar o efeito da aplicação de doses de selenato e selenito na biofortificação de arroz (Oryza sativa) com Se, bem como a influência dessas formas de Se nos teores de P, S, Fe e Zn nos grãos.

\section{Material e Métodos}

O experimento foi conduzido em casa de vegetação no Departamento de Ciência do Solo, da Universidade Federal de Lavras, MG, em vasos com $4 \mathrm{dm}^{3}$ de solo, preenchidos com amostras da camada de 0-20 cm de um Latossolo Vermelho-Amarelo distrófico, de textura média. As análises físicas e químicas do solo, conforme Claessen (1997), e mineralógicas, de acordo com Souza (2005), indicaram: $60 \mathrm{dag} \mathrm{kg}^{-1} \mathrm{de}$ areia; 17 dag kg ${ }^{-1}$ de silte; 23 dag $\mathrm{kg}^{-1}$ de argila; $\mathrm{pH}$ em água de 5,4; 1,2 dag $\mathrm{kg}^{-1}$ de MOS; P (Mehlich-1) de $0,9 \mathrm{mg} \mathrm{dm}^{-3} ; 23,4 \mathrm{mg} \mathrm{dm}^{-3}$ de K; 0,3 $\mathrm{cmol}_{\mathrm{c}} \mathrm{dm}^{-3}$ de Ca; 0,1 cmol $_{\mathrm{c}} \mathrm{dm}^{-3}$ de $\mathrm{Mg} ; 0,1 \mathrm{cmol}_{\mathrm{c}} \mathrm{dm}^{-3}$ de Al; 0,9 cmol $_{\mathrm{c}} \mathrm{dm}^{-3}$ de $\mathrm{H}+\mathrm{Al} ; 20,5 \mathrm{mg} \mathrm{L}^{-1}$ de P-rem; $37,8 \mathrm{~g} \mathrm{~kg}^{-1}$ de $\mathrm{Fe}_{2} \mathrm{O}_{3}$; e $168,1 \mathrm{~g} \mathrm{~kg}^{-1} \mathrm{de} \mathrm{Al}_{2} \mathrm{O}_{3}$. O teor total de Se foi de $0,064 \mathrm{mg} \mathrm{dm}^{-3}$, conforme Ramos et al. (2010).

Utilizou-se o delineamento experimental inteiramente casualizado, em arranjo fatorial $5 \times 2$, com cinco doses de $\mathrm{Se}\left(0,0,75,1,50,3,0\right.$ e $\left.6,0 \mathrm{mg} \mathrm{dm}^{-3}\right)$ aplicadas no solo, e duas formas de $\mathrm{Se}$ (selenato de sódio $\mathrm{Na}_{2} \mathrm{SeO}_{4}$ - e selenito de sódio $-\mathrm{Na}_{2} \mathrm{SeO}_{3}$,), com cinco repetições, o que totalizou 50 parcelas. Cada unidade experimental foi constituída por duas plantas por vaso.

Com base na análise química do solo, foi efetuada a calagem para elevar a saturação por bases a $50 \%$, com uso de calcário calcinado e micropulverizado, que continha $32 \%$ de $\mathrm{CaO}, 15 \%$ de $\mathrm{MgO}$ e PRNT de $94,5 \%$. Após incubação do solo por 30 dias, com umidade próxima a $60 \%$ do volume total de poros (VTP), as doses de selênio foram aplicadas ao solo, em ambas as formas. Juntamente com a aplicação dos tratamentos com Se, foi realizada a adubação básica de plantio com $80 \mathrm{mg}$ de N, $250 \mathrm{mg}$ de $\mathrm{P}, 70 \mathrm{mg}$ de $\mathrm{K}$ e $60 \mathrm{mg}$ de S por $\mathrm{dm}^{3}$ de solo, como nitrato de amônio $\left(\mathrm{NH}_{4} \mathrm{NO}_{3}\right)$, fosfato monoamônio $\left(\mathrm{NH}_{4} \mathrm{H}_{2} \mathrm{PO}_{4}\right)$ e sulfato de potássio $\left(\mathrm{K}_{2} \mathrm{SO}_{4}\right)$. A adubação básica com micronutrientes consistiu na aplicação de 3,6 mg de $\mathrm{Mn} ; 1,5 \mathrm{mg}$ de $\mathrm{Cu}$; 5,0 mg de Zn; 0,5 mg de B; e 0,15 mg de Mo por $\mathrm{dm}^{3}$ de solo, fornecidos na forma de cloreto de manganês $\left(\mathrm{MnCl}_{2} \cdot 4 \mathrm{H}_{2} \mathrm{O}\right)$, sulfato de cobre $\left(\mathrm{CuSO}_{4} \cdot 5 \mathrm{H}_{2} \mathrm{O}\right)$, sulfato de zinco $\left(\mathrm{ZnSO}_{4} .7 \mathrm{H}_{2} \mathrm{O}\right)$, ácido bórico $\left(\mathrm{H}_{3} \mathrm{BO}_{3}\right)$ e molibdato de amônio $\left[\left(\mathrm{NH}_{4}\right)_{6} \mathrm{Mo}_{7} \mathrm{O}_{24} \cdot 4 \mathrm{H}_{2} \mathrm{O}\right]$.

Em seguida, foram semeadas dez sementes de arroz (Oryza sativa L. cultivar BRSMG Relâmpago) por vaso e desbastadas para duas plântulas uma semana após emergência. Durante o período de cultivo, o arroz recebeu adubações de cobertura nas doses de $450 \mathrm{mg}$ de $\mathrm{N}$ e $350 \mathrm{mg}$ de $\mathrm{K}$ por $\mathrm{dm}^{3}$ de solo, divididas em oito aplicações, com nitrato de amônio $\left(\mathrm{NH}_{4} \mathrm{NO}_{3}\right)$ e nitrato de potássio $\left(\mathrm{KNO}_{3}\right)$. Durante a condução do experimento, a umidade do solo foi mantida próxima à capacidade de campo por meio de pesagens diárias do conjunto vaso-solo-planta, repondo-se o volume evapotranspirado com água deionizada.

A colheita das plantas foi feita no final do ciclo. O material vegetal foi seco em estufa com circulação forçada de ar a $65-70^{\circ} \mathrm{C}$, para quantificação da massa seca de raiz, de parte aérea (folhas e caule) e de grãos. Foram realizadas, nos grãos, análises químicas para a determinação dos teores de P, S, Fe e Zn, de acordo com Malavolta et al. (1997).

Para a determinação dos teores de Se nas raízes, na parte aérea e nos grãos, realizou-se a digestão das amostras conforme Ramos et al. (2010). A quantificação de Se nos extratos foi realizada por espectrometria de fluorescência de raios-X por reflexão total utilizando o S2 PICOFOX (Bruker, Berlin, Alemanha), com adição de $10 \mu \mathrm{L}$ do extrato em porta-amostras de quartzo. Utilizou-se o gálio como padrão interno, e o 
tempo de leitura foi de $400 \mathrm{~s}$. Para avaliar a acurácia das análises, utilizou-se material certificado White Clover (BCR402, Institute for Reference Materials and Measurements, Geel, Bélgica), o qual foi incluído em cada bateria de digestão. Os teores de Se foram relacionados à massa seca produzida. Determinou-se o acúmulo de Se em cada parte e no total da planta, para cada tratamento. Com os dados obtidos, estimaramse os índices: eficiência das raízes na absorção de Se (mg de Se total acumulado na planta/g de massa seca de raízes) e eficiência de aproveitamento do Se aplicado $100[(\mathrm{mg}$ de Se total acumulado)/(mg de Se aplicado)].

Os dados obtidos foram submetidos à análise de variância, ao teste de média (Scott-Knott) e à regressão polinomial com uso do programa estatístico SISVAR (Ferreira, 2008).

\section{Resultados e Discussão}

A produção de matéria seca da raiz e da parte aérea foi afetada significativamente pela interação entre doses e formas de Se (Figura 1). Para a produção de grãos, essa interação não foi significativa. No entanto, essa variável apresentou diferença significativa quando se comparou as formas de Se utilizadas.

Para a massa seca das raízes (Figura 1), o aumento das doses de selenato proporcionou redução linear, enquanto o de selenito, quadrática. Houve redução na produção de raízes, ao se comparar as maiores doses de Se com o tratamento controle, de aproximadamente 13 e $21 \%$, para o selenito e o selenato, respectivamente. Esse comportamento diferenciado promovido pelas formas de Se aplicadas pode ser atribuído à adsorção do selenito no solo, o que tornou esse elemento menos disponível e menos fitotóxico para as raízes, em comparação ao selenato. No presente trabalho, o solo, apesar de apresentar textura média (23\% de argila) e baixos teores de óxidos de $\mathrm{Fe}$ e $\mathrm{Al}$ (37,8 e 168,1 $\mathrm{g} \mathrm{kg}^{-1}$, respectivamente), provavelmente influenciou a disponibilidade do Se quando se utilizou o selenito. Segundo Hopper \& Parker (1999), as diferenças de comportamento entre o selenato e o selenito nos solos são esperadas, em razão das suas diferentes afinidades com os coloides do solo, o que afeta, consequentemente, a sua solubilidade e disponibilidade para as plantas. Esses autores relataram que, para uma mesma quantidade de selenato e selenito aplicada no solo, o selenito é mais adsorvido nas superfícies dos óxidos, o que o torna menos disponível para as plantas.

A produção da massa seca da parte aérea (Figura 1) apresentou resposta crescente até a dose de $0,75 \mathrm{mg} \mathrm{dm}^{-3}$ de selenato, a qual proporcionou aumento de $8 \%$ quando o selenato foi aplicado. Contudo, o aumento das doses para ambas as formas de Se proporcionou queda na produção para essa variável.
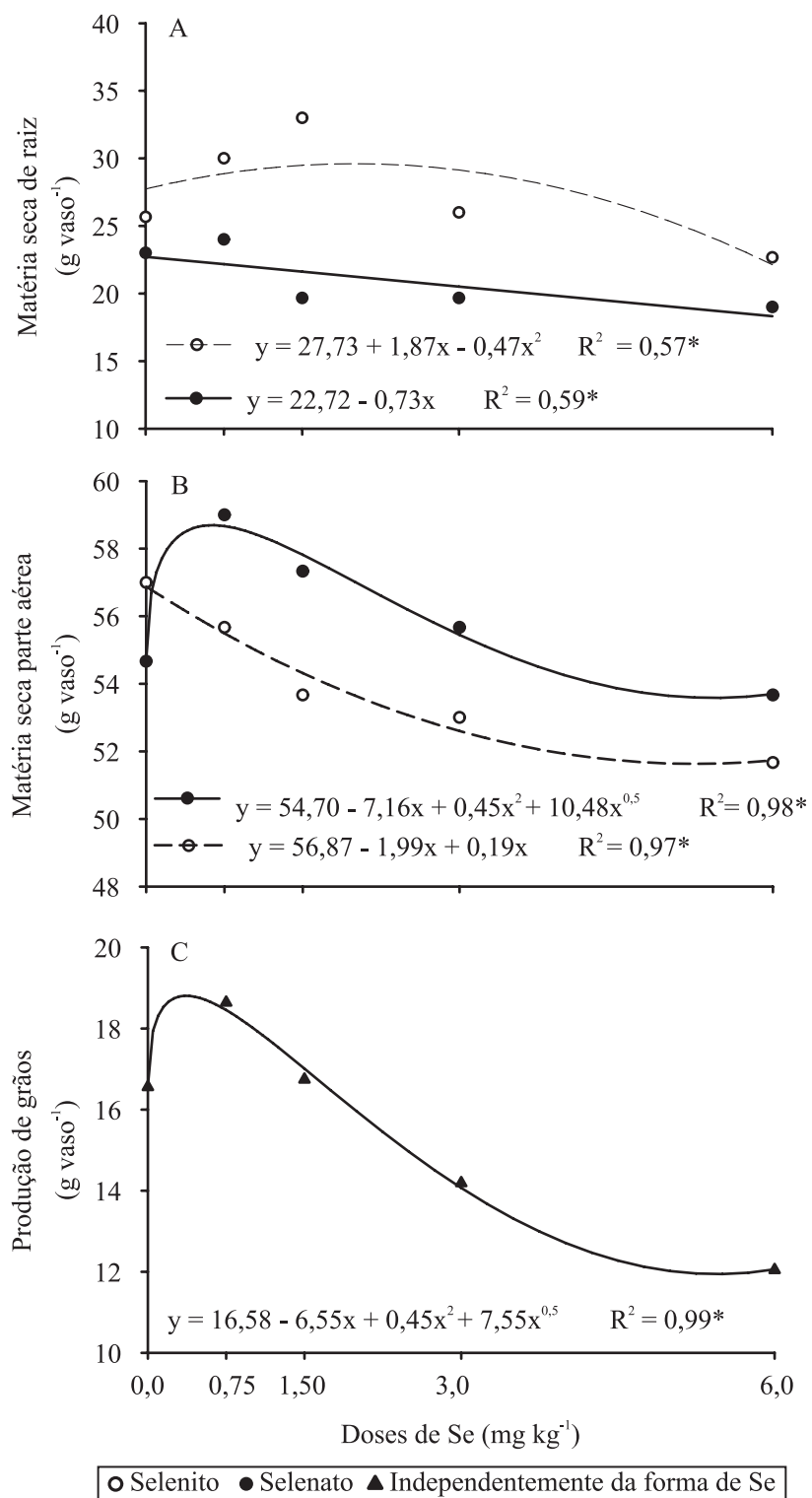

Figura 1. Massa seca da raiz (A), parte aérea (B) e produção de grãos (C) em plantas de arroz em função da aplicação de doses e formas de Se. "Significativo pelo teste t, a 5\% de probabilidade. 
O efeito da aplicação de Se para a produção de grãos seguiu a mesma tendência apresentada para a produção de massa seca da parte aérea quando se aplicou o selenato (Figura 1), ou seja, houve aumento de $13 \%$ na produção de grãos na dose de $0,75 \mathrm{mg} \mathrm{dm}^{-3}$, independentemente das formas de Se aplicadas. Quanto às formas de Se, observou-se que o selenito foi superior em $11 \%$, em comparação ao selenato, na produção de grãos. Os aumentos na produção da parte aérea e de grãos eram esperados, uma vez que o Se aplicado em baixas concentrações aumenta a atividade antioxidante, o que leva a uma maior produção vegetal (Hartikainen et al., 2000; Ramos et al., 2011).

Embora o selenito tenha proporcionado maior produção de matéria seca de raiz, esse fato não se repetiu na eficiência das raízes na absorção de Se (Figura 2). Assim, o total de Se acumulado na planta por grama de raiz produzida, em todas as doses aplicadas, foi sempre superior quando se utilizou o selenato. Esse fato está
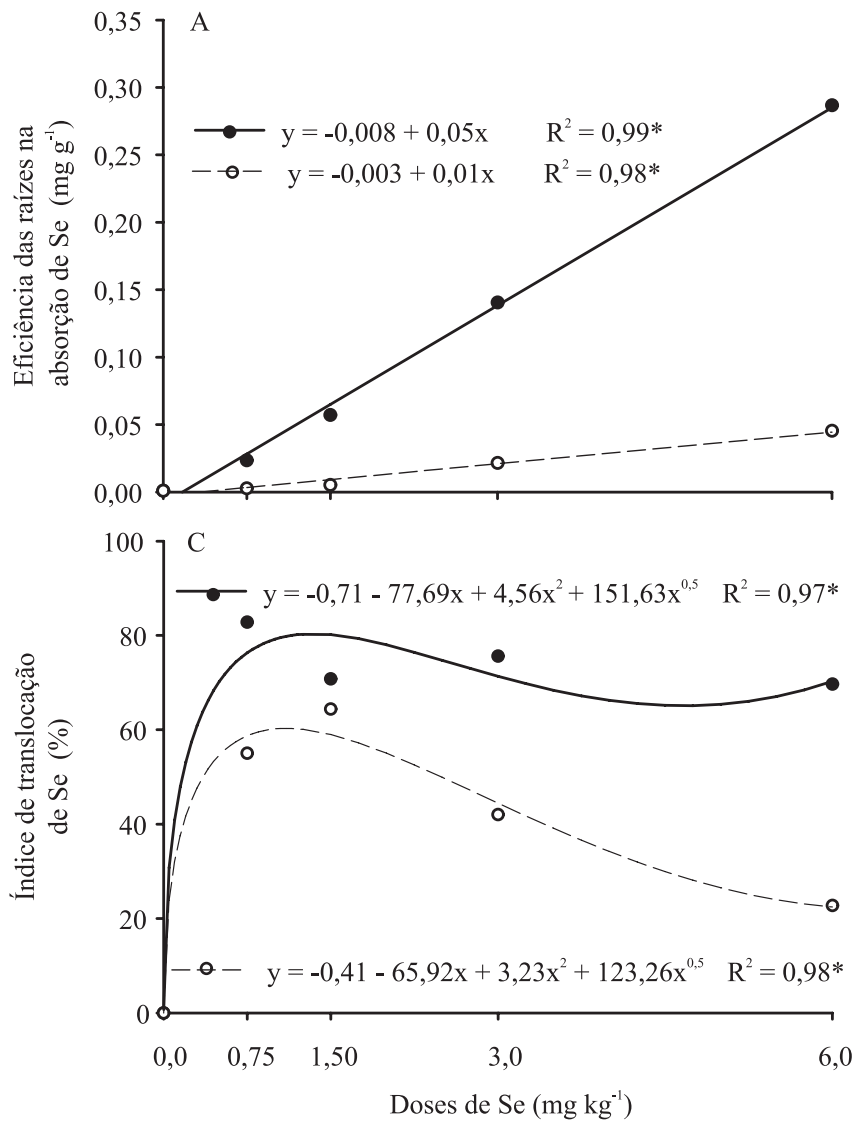

relacionado ao maior teor de Se nos tecidos e, também, ao maior acúmulo de Se nas plantas de arroz quando o selenato foi aplicado.

Com relação à eficiência no aproveitamento do Se aplicado (Figura 2), o selenato, como observado para a eficiência das raízes na absorção de Se, proporcionou melhor aproveitamento do Se aplicado. Wijnja \& Schulthess (2000) observaram que o comportamento do selenato no solo é semelhante ao do sulfato, enquanto o selenito se assemelha mais ao fosfato. Portanto, em solos altamente intemperizados, os óxidos de $\mathrm{Fe}, \mathrm{Al}$ e $\mathrm{Mn}$ podem diminuir consideravelmente o aproveitamento do $\mathrm{Se}$ quando se utiliza o selenito.

Verificou-se que a translocação do Se foi sempre maior quando fornecido na forma de selenato (Figura 2). Nos tratamentos que receberam as doses de Se, em média, 78 e $47 \%$ do elemento aplicado foi translocado para a parte aérea, para o selenato e
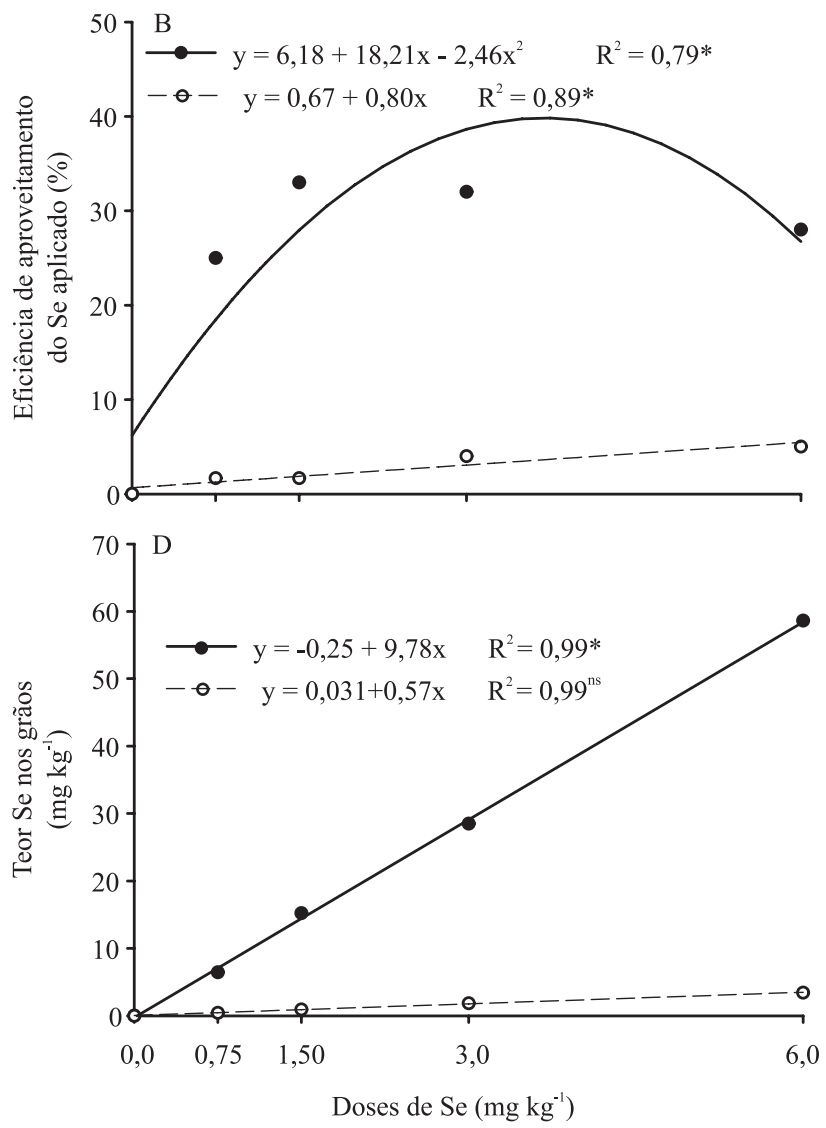

o Selenito • Selenato

Figura 2. Eficiência das raízes na absorção de Se (A), eficiência no aproveitamento do Se aplicado (B), índice de translocação de Se (C) e teor de Se nos grãos (D) em plantas de arroz em função da aplicação de doses e formas de Se. "Significativo pelo

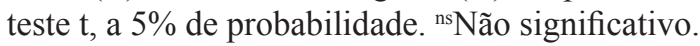


o selenito, respectivamente. De acordo com Li et al. (2008), a superioridade do selenato sobre o selenito no transporte de Se pelas plantas decorre da menor conversão do selenato a formas orgânicas de Se nas raízes, o que favorece a mobilidade no xilema. Já o selenito, quando absorvido pelas plantas, é rapidamente convertido para as formas orgânicas, as quais apresentam menor mobilidade no xilema. Esses resultados são semelhantes aos obtidos em outros trabalhos, os quais relatam superioridade do selenato sobre o selenito no transporte de Se pelas plantas (Ramos et al., 2010; Sharma et al., 2010).

Os teores de Se nos grãos de arroz foram afetados significativamente pela interação entre doses e formas de Se (Figura 2). Os maiores teores desse elemento foram observados quando se utilizou o Se na forma de selenato. Esse fato provavelmente resulta da maior absorção das raízes, do melhor aproveitamento pela planta, da translocação do Se para a parte aérea e da maior eficiência em redistribuir esse elemento para
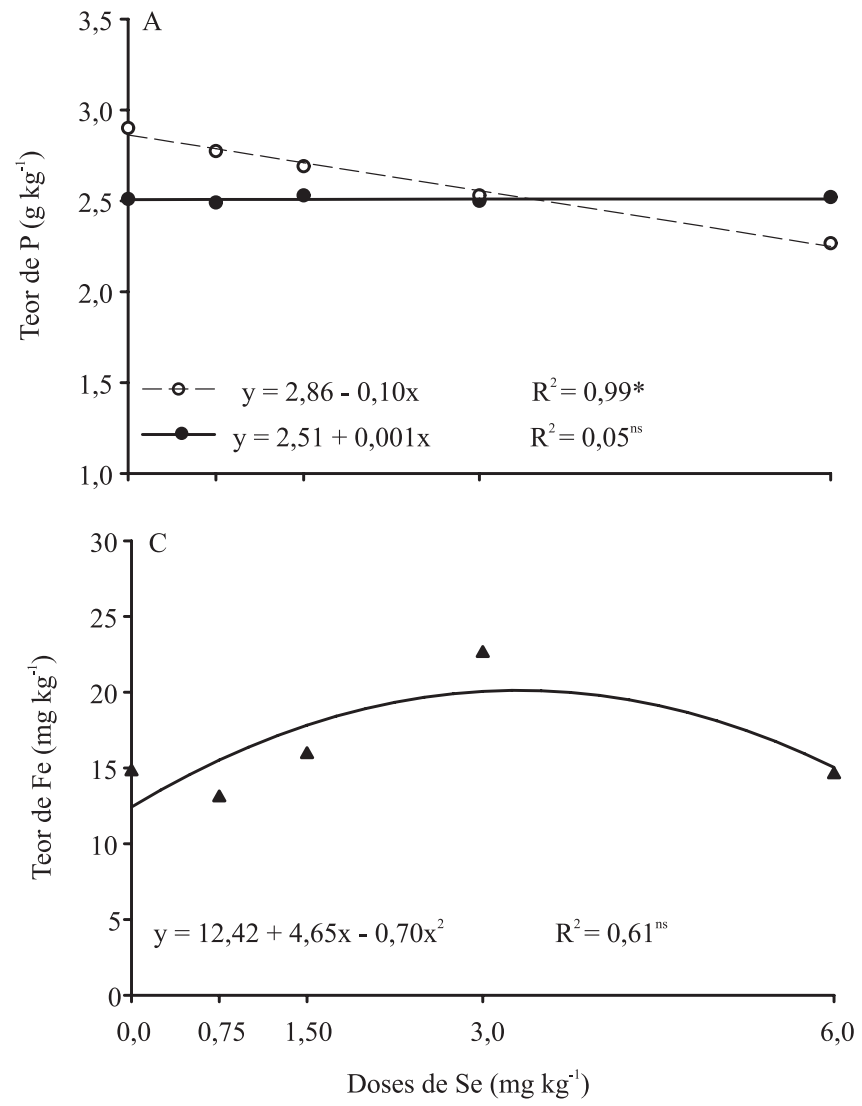

os grãos, ao se utilizar o selenato. Esse resultado corrobora os de Li et al. (2010), que avaliaram o efeito de diferentes manejos da cultura e formas de Se nos teores desse elemento nos grãos de arroz.

De acordo com o United States Department of Agriculture (2003), a recomendação de ingestão diária mínima de Se para adultos é de $70 \mu \mathrm{g}$ por dia e o nível máximo tolerável é de $400 \mu$ g por dia. No presente trabalho, o teor de Se encontrado nos grãos de arroz pode contribuir para a ingestão diária de Se em, aproximadamente, 1.100 e $80 \mu \mathrm{g}$ por dia ao se utilizar o selenato e o selenito, respectivamente, na dose de $0,75 \mathrm{mg} \mathrm{dm}^{-3}$. Porém, ainda são necessários mais estudos a respeito da biofortificação agronômica com Se em arroz, para a adequação das doses a serem aplicadas, para maior segurança alimentar.

Os teores de $\mathrm{P}$ e $\mathrm{S}$ nos grãos foram afetados significativamente pelas doses e pelas formas de $\mathrm{Se}$ (Figura 3). Observou-se que as doses de selenato não influenciaram o teor de $\mathrm{P}$ nos grãos, enquanto $\mathrm{o}$
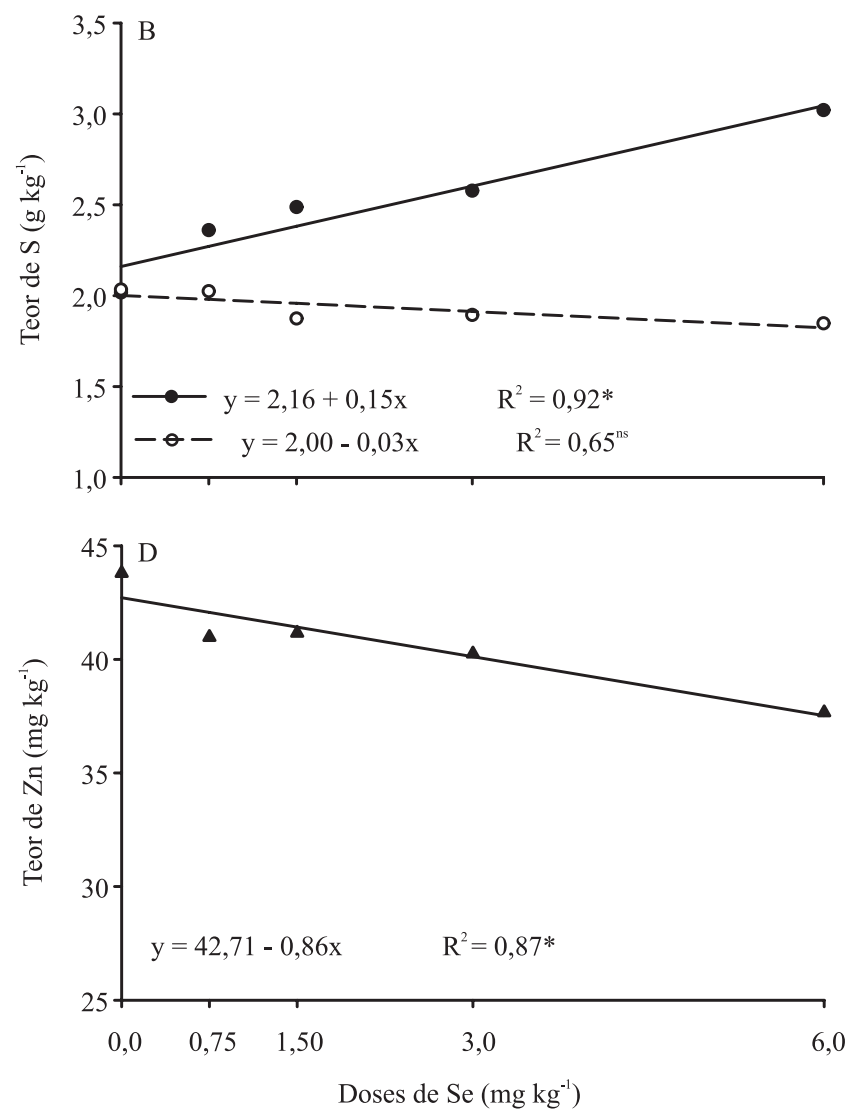

o Selenito $\bullet$ Selenato $\Delta$ Independentemente da forma de Se

Figura 3. Teor de $\mathrm{P}(\mathrm{A}), \mathrm{S}$ (B), Fe (C) e Zn (D) em grãos de arroz em função da aplicação de doses e formas de Se. "Significativo

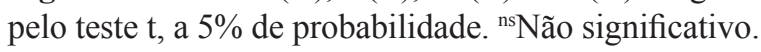


selenito proporcionou redução. Nesse sentido, estudos têm mostrado que o selenito compete com o P no processo de absorção (Hopper \& Parker, 1999) e, como consequência, afeta o teor de P nas plantas ( $\mathrm{Li}$ et al., 2008).

Verificou-se que o aumento nas doses de selenato incrementou o teor de S nos grãos de arroz. Entretanto, não houve efeito significativo quando se utilizou o Se na forma de selenito. Ramos et al. (2011), ao avaliar o efeito do selenito e do selenato em diversos germoplasmas de alface, observaram que o selenato promoveu aumento no teor de S. Resultados semelhantes aos do presente trabalho também foram verificados por Mikkelsen \& Wan (1990) em cevada e arroz. Segundo esses autores, a interação sinérgica entre o selenato e o sulfato pode ocorrer em diversas espécies vegetais.

Os teores de $\mathrm{Fe}$ e $\mathrm{Zn}$ não foram influenciados significativamente pelas formas de Se, e, no geral, o aumento nas doses de Se não influenciou o teor de $\mathrm{Fe}$ (Figura 3), mas diminuiu o de Zn. Contudo, Zembala et al. (2010), ao avaliar colza e trigo, relataram que a aplicação do Se aumentou os teores de $\mathrm{Zn}$, mas diminuiu os de Fe.

\section{Conclusões}

1. O selênio aplicado na forma de selenato nas plantas de arroz proporciona maior eficiência na absorção desse elemento pelas raízes, no aproveitamento pela planta, na translocação para a parte aérea, bem como maior teor nos grãos.

2. A aplicação do Se na adubação altera os teores de $\mathrm{P}, \mathrm{S}$ e $\mathrm{Zn}$, mas não afeta os de Fe nos grãos de arroz.

\section{Agradecimentos}

À Fundação deAmparoà Pesquisa do Estado deMinas Gerais, ao Conselho Nacional de Desenvolvimento Científico e Tecnológico e à Coordenação de Aperfeiçoamento de Pessoal de Nível Superior, pelo apoio financeiro e pela concessão de bolsa de estudo.

\section{Referências}

CHEN, L.; YANG, F.; XU, J.; YUN, H.; HU, Q.; ZHANG, Y.; PAN, G. Determination of selenium concentration of rice in China and effect of fertilization of selenite and selenate on selenium content of rice. Journal of Agricultural and Food Chemistry, v.50, p.5128-5130, 2002.
CLAESSEN, M.E.C. (Org.). Manual de métodos de análise de solo. 2.ed. rev. atual. Rio de Janeiro: Embrapa-CNPS, 1997. 212p. (Embrapa-CNPS. Documentos, 1).

COMBS, G.F. Selenium in global food systems. British Journal of Nutrition, v.85, p.517-547, 2001.

FERREIRA, D.F. SISVAR: um programa para análises e ensino de estatística. Revista Symposium, v.6, p.36-41, 2008.

FERREIRA, K.S.; GOMES, J.C.; BELLATO, C.R.; JORDÃO, C.P. Concentrações de selênio em alimentos consumidos no Brasil. Revista Panamericana de Salud Pública, v.11, p.172-177, 2002.

GRAHAM, R.D.; WELCH, R.M.; SAUNDERS, D.A.; ORTIZ-MONASTERIO, I.; BOUIS, H.E.; BONIERBALE, M.; DE HAAN, S.; BURGOS, G.; THIELE, G.; LIRIA, R. Nutritious subsistence food systems. Advances in Agronomy, v.92, p.1-74, 2007.

HARTIKAINEN, H.; XUE, T.L.; PIIRONEN, V. Selenium as an anti-oxidant and pro-oxidant in ryegrass. Plant and Soil, v.225, p.193-200, 2000 .

HOPPER, J.L.; PARKER, D.R. Plant availability of selenite and selenate as influenced by the competing ions phosphate and sulfate. Plant and Soil, v.210, p.199-207, 1999.

LI, H.-F.; LOMBI, E.; STROUD, J.L.; MACGRATH, S.P.; ZHAO, F. Selenium speciation in soil and rice: influence of water management and Se fertilization. Journal of Agricultural and Food Chemistry, v.58, p.11837-11843, 2010.

LI, H.-F.; MCGRATH, S.P.; ZHAO, F.-J. Selenium uptake, translocation and speciation in wheat supplied with selenate or selenite. New Phytologist, v.178, p.92-102, 2008.

LUCCA, P.; POLETTI, S.; SAUTTER, C. Genetic engineering approaches to enrich rice with iron and vitamin A. Physiologia Plantarum, v.126, p.291-303, 2006.

MAIHARA, V.A.; GONZAGA, I.B.; SILVA, V.L.; FÁVARO, D.I.T.; VASCONCELLOS, M.B.A.; COZZOLINO, S.M.F. Daily dietary selenium intake of selected Brazilian population groups. Journal of Radioanalytical and Nuclear Chemistry, v.259, p.465-468, 2004.

MALAVOLTA, E.; VITTI, G.C.; OLIVEIRA, S.A. de. Avaliação do estado nutricional das plantas: princípios e aplicações. 2.ed. Piracicaba: Potafos, 1997. 319p.

MIKKELSEN, R.L.; WAN, H.F. The effect of selenium on sulfur uptake by barley and rice. Plant and Soil, v.121, p.151-153, 1990.

RAMOS S.J.; FAQUIN, V.; GUILHERME, L.R.G.; CASTRO, E.M.; AVILA, F.W.; CARVALHO, G.S.; BASTOS, C.E.A.; OLIVEIRA, C. Selenium biofortification and antioxidant activity in lettuce plants fed with selenate and selenite. Plant Soil and Environment, v.56, p.584-588, 2010.

RAMOS, S.J.; RUTZKE, M.A.; HAYNES, R.J.; FAQUIN, V.; GUILHERME, L.R.G.; LI, L. Selenium accumulation in lettuce germplasm. Planta, v.233, p.649-660, 2011.

RAYMAN, M.P. The argument for increasing selenium intake. Proceedings of the Nutrition Society, v.61, p.203-215, 2002.

ROVIRA, M.; GIMÉNEZ, J.; MARTÍNEZ, M.; MARTÍNEZ-LLADÓ, X.; PABLO, J.; MARTÍ, V.; DURO, L. 
Sorption of selenium (IV) and selenium (VI) onto natural iron oxides: goethite and hematite. Journal of Hazardous Materials, v.150, p.279-284, 2008.

SHARMA, S.; BANSAL, A.; DHILLON, S.; DHILLON, K. Comparative effects of selenate and selenite on growth and biochemical composition of rapeseed (Brassica napus L.). Plant and Soil, v.329, p.339-348, 2010.

SOUZA, R.F. Dinâmica de fósforo em solos sob influência da calagem e adubação orgânica, cultivados com feijoeiro. 2005. 141p. Tese (Doutorado) - Universidade Federal de Lavras, Lavras.

UNITED STATES DEPARTMENT OF AGRICULTURE. Dietary reference intakes: elements. 2003. Available at: $<$ http://www.iom. edu/Global/News\%20Announcements/ /media/48FAAA2FD9E7 4D95BBDA2236E7387B49. ashx>. Accessed on: 18 Jan. 2010.

WHITE, P.J.; BROADLEY, M.R. Biofortification of crops with seven mineral elements often lacking in human diets - iron, zinc, copper, calcium, magnesium, selenium and iodine. New Phytologist, v.182, p.49-84, 2009.

WIJNJA, H.; SCHULTHESS, C.P. Vibrational spectroscopy of selenate and sulfate adsorption mechanisms on Fe and Al (hydr) oxide surfaces. Journal of Colloid and Interface Science, v.229, p.286-297, 2000.

ZEMBALA, M.; FILEK, M.; WALAS, S.; MROWIEC, H.; KORNAS, A.; MISZALSKI, Z.; HARTIKAINEN, H. Effect of selenium on macro- and microelement distribution and physiological parameters of rape and wheat seedlings exposed to cadmium stress. Plant and Soil, v.329, p.457-468, 2010.

ZHANG, P.C.; SPARKS, D.L. Kinetics of selenate and selenite adsorption/desorptionat the goethite/waterinterface. Environmental Science and Technology, v.24, p.1848-1856, 1990.

ZHANG, Y.L.; PAN, G.X.; CHEN, J.; HU, Q. Uptake and transport of selenite and selenate by soybean seedlings of two genotypes. Plant and Soil, v.253, p.437-443, 2003.

Recebido em 4 de outubro de 2011 e aprovado em 30 de abril de 2012 